\title{
Genetic analysis of grain shape and weight after cutting rice husk
}

\author{
J.R. Fu' ${ }^{1,2,3 *}$, L.X. Zhu ${ }^{1,2 *}$, X.T. Sun ${ }^{1,2,3}$, D.H. Zhou ${ }^{1,2}$, L.J. Ouyang ${ }^{1,2,3}$, J.M. Bian ${ }^{1,2,3}$, \\ H.H. $\mathrm{He}^{1,2,3}$ and J. $\mathrm{Xu}^{1,2}$ \\ 1Jiangxi Agricultural University, Nanchang, China \\ ${ }^{2}$ Key Laboratory of Crop Physiology, Ecology and Genetic Breeding of Jiangxi Province, \\ Nanchang, China \\ ${ }^{3}$ Southern Regional Collaborative Innovation Center for Grain and Oil Crops, \\ Hunan Agricultural University, Changsha, China \\ *These authors contributed equally to this study. \\ Corresponding authors: H.H. He / J. Xu \\ E-mail: hhhua64@163.com / xujie198615@foxmail.com \\ Genet. Mol. Res. 14 (4): 17739-17748 (2015) \\ Received August 24, 2015 \\ Accepted October 8, 2015 \\ Published December 21, 2015 \\ DOI http://dx.doi.org/10.4238/2015.December.21.47
}

ABSTRACT. Grain shape and weight are the most important components of rice yield and are controlled by quantitative trait loci (QTLs). In this study, a double-haploid population, derived from the cross of japonica CJ06 and indica TN1, was used to analyze QTLs for grain shape and weight under two conditions: normal growth with unbroken husk and removing partial husk after flowering. Correlation analysis revealed that these traits, except grain weight, had a connection between the two conditions. Twenty-nine QTLs for grain shape and weight were detected on chromosomes 1 to 3; 6; 8 to 10 ; and 12, with the likelihood of odds value ranging from 2.38 to 5.36 , including 10 different intervals. Some intervals were specifically detected after removing partial husk. The results contribute to the understanding of the genetic basis of grain filling and growth regulation, and provide us some assistance for improving grain plumpness in rice breeding.

Key words: Quantitative trait loci; Grain shape; Grain weight; Rice husk 


\section{INTRODUCTION}

Rice is one of the most important cereal crops in the world and for many years, increasing yield has been set as the overriding objective of breeding (Miura et al., 2011). Rice yield is determined by three major components: number of panicles per plant, number of grains per panicle, and grain weight (Song et al., 2007). Among them, the most reliable trait is grain weight, which is measured as the 1000-grain weight (KGW). Grain size and filling are important for rice yield potential, mainly for KGW (Takeda and Matsuoka, 2008). Grain shape is composed of grain length, width, and thickness, which are positively correlated with grain weight (Tan et al., 2000). Grain shape is an important factor for market values of rice grain products (Huang et al., 2013). Long and slender grains are generally preferred in Southern China, the USA, Europe, and Southeast Asian countries, whereas short and round grains are preferred in Japan, Korea, and Northern China (Unnevehr et al., 1992).

To date, 13 genes associated with grain shape and grain weight have been cloned; these can be divided into three groups on the basis of mutant phenotypes. The first group comprises dwarf1 $(D 1), D 2, D 11$, and D61. Mutants resulted by those genes showed dwarf plants and detrimental pleiotropic effects on organ growth, including a reduction in seed size (Ashikari et al., 1999; Yamamuro et al., 2000; Hong et al., 2003; Tanabe et al., 2005). The second group appears to specifically affect grain traits: GS3 plays important roles in grain length and grain weight (Mao et al., 2010); GW2, GW5, GS5, and GW8/SPL16 affect grain width and weight (Song et al., 2007; Weng et al., 2008; Li et al., 2011; Wang et al., 2012), and GIF1 is involved in grainfilling (Wang et al., 2008). The third group includes the small and round seed genes (SRS). Mutations in SRS1, SRS3, and SRS5 affect cell length of the rice flower lemma (Kitagawa et al., 2010; Li et al., 2010; Segami et al., 2012). In the past 20 years, after the development of DNA markers and high-density marker linkage maps in rice, quantitative trait locus (QTL) mapping was introduced as a method for understanding molecular genetic mechanisms of rice grain shape and weight. More than 400 QTLs for rice grain shape and weight have been detected and mapped on chromosomes. Over 167 QTLs for KGW, 103 for grain length, and 95 for grain width were detected in previous studies with different parents and populations (Huang et al., 2013). The cloned genes described above are located in those QTLs.

Rice grain is composed of husk and brown rice. Grain size and weight are determined by both the storage capacity of the husk (sink), and supplying capacity of filling substance (source) (Ma et al., 2009). Rice husk is considered the container of filling, and several studies have indicated that the volume of hulled rice was closely related to that of paddy, the length, width, thickness, volume, and weight of grains were increased with the increment of husk size (Wang et al., 1995; Wang et al., 1998). In another hand, the husk size would limit grain filling because the sink capacity is not enough for source (Zhou et al., 2002). The third group of mutants described above showed short lemma length, resulting in small and round grain.

In this study, we cut partial husk at the filling stage, to eliminate the limitation of husk size on filling. We then attempted to characterize the genetic basis of grain shape and weight, by analyzing the QTLs of main and epistatic effects both with unbroken husk and removed husk at filling stage, using a population of doubled haploid (DH) lines, derived from a cross between CJ06 and TN1.

\section{MATERIAL AND METHODS}

\section{Plant material}

A DH population consisting of 120 lines was developed via an anther culture of an F1 
hybrid between typical japonica CJ06 and indica TN1 rice cultivars. The two parents and all DH lines seeds were grown with a planting density of $20 \times 20 \mathrm{~cm}$ in the experimental farm of the Jiangxi Agricultural University, Nangchang, during rice growing seasons in 2010. Two parents and each $\mathrm{DH}$ line were applied with three replications. Each $\mathrm{DH}$ line was planted in four rows, with six plants in each row. The plants in the middle were harvested individually to score grain length, width, and KGW.

\section{Measurement of traits}

The study was carried out under two conditions: normal growth with unbroken husk and removing partial husk after flowering, and bagged the panicles. Approximately 40 days after flowering, rice grains were harvested, air-dried, and brown rice obtained. Grain length and width were calculated by vernier caliper. Grains were weighed to obtain KGW using an electronic scale with three replications.

\section{Data and QTL analysis}

Population distribution and correlation analysis were performed using the SAS8.0 statistical software (SAS Institute, Cary, NC, USA). The genetic linkage map was constructed using a total of 178 simple sequence repeat (SSR) and sequence tagged site (STS) markers distributed evenly on all 12 rice chromosomes, as previously described (Yang et al., 2014). The genetic linkage map spanned $1674.8 \mathrm{cM}$, with the average interval of $9.4 \mathrm{cM}$ between the two adjacent markers.

The QTLs were detected by interval mapping using the QTLMAPPER 1.6 software, which was developed based on the mixed linear model approach (Wang et al., 1999). A likelihood of odds (LOD) threshold of 2.5 was used to declare the presence of a putative QTL in a given genomic region. Genetic parameters, additive effects, and accounted variation of each QTL were also estimated. The relative contribution of a genetic component was calculated as the proportion of phenotypic variance explained by the component in the selected model (McCouch, 2008).

\section{RESULTS}

\section{Phenotypic variation in parents and DH lines}

Grain shape and weight for parents (CJ06 and TN1) and the DH population are summarized in Table 1. Without cutting the husk, grain length, width and weight did not differ significantly between the two parents. However, after removing the husk, there was a large difference between parents in grain shape and weight, especially in grain length and weight. The grain length in cutting husk (GLC) was higher in TN1 $(7.773 \mathrm{~mm})$ than CJ06 $(7.207 \mathrm{~mm})$; a similar observation was made for the 1000-grain weight in cutting husk (KGWC). However, a relatively small difference in both grain length (GL) and 1000-grain weight (KGW) were observed between the parents without cutting the husk. For example, the value of GL in CJ06 and TN1 was $5.517 \mathrm{~mm}$ and $5.697 \mathrm{~mm}$, respectively. These results indicated that changes in the grain shape and weight with and without the treatment were larger in TN1 than in CJ06. After cutting the husk, the grain length of CJ06 increased by $30.6 \%$, while it was $36.5 \%$ in TN1; the grain weight was up to 7 and $25.7 \%$ for CJ06 and TN1, respectively. All detected traits showed a continuous distribution in the $\mathrm{DH}$ population, and transgressive variation was also seen in the $\mathrm{DH}$ population (Table 1; Figure 1). 
Table 1. Determination of grain shape and weight in the doubled haploid population and their parents.

\begin{tabular}{lcccc}
\hline Traits & \multicolumn{2}{c}{ Parent } & & \multicolumn{2}{c}{ Doubled haploid population } \\
\cline { 2 - 3 } & CJ06 & TN1 & Mean \pm SD & Range \\
\hline Grain length (GL; mm) & 5.517 & 5.697 & $5.115 \pm 0.765$ & $3.2-6.153$ \\
Grain width (GW; mm) & 2.780 & 2.897 & $2.511 \pm 0.587$ & $1.45-4.853$ \\
1000-grain weight (KGW; g) & 22.933 & 23.633 & $18.1 \pm 3.81$ & $11.4-24.3$ \\
Grain length in cutting husk (GLC; mm) & 7.207 & 7.773 & $6.433 \pm 0.964$ & $4.03-8.03$ \\
Grain width in cutting husk (GWC; mm) & 2.193 & 2.297 & $2.073 \pm 0.252$ & $1.27-2.447$ \\
1000-grain weight in cutting husk (KGWC; g) & 24.533 & 29.700 & $20.3 \pm 4.762$ & $11.1-33.7$ \\
\hline
\end{tabular}

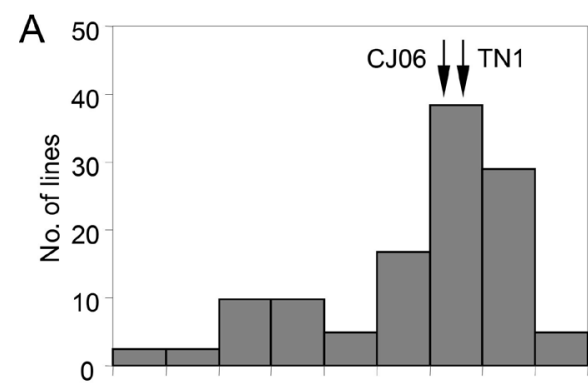

$\begin{array}{lllllllll}3.2 & 3.6 & 4.0 & 4.4 & 4.8 & 5.2 & 5.6 & 6.0 & 6.4\end{array}$ Grain length $(\mathrm{mm})$
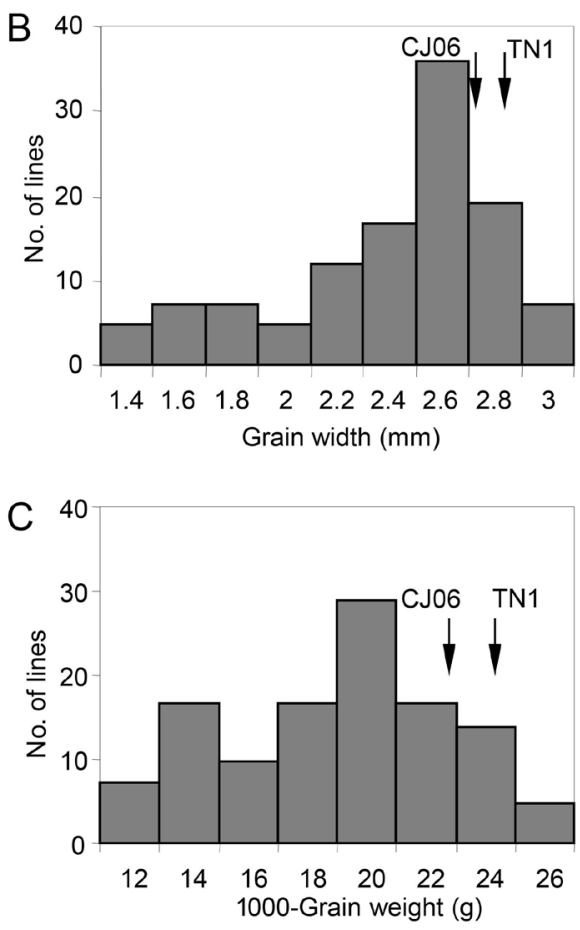

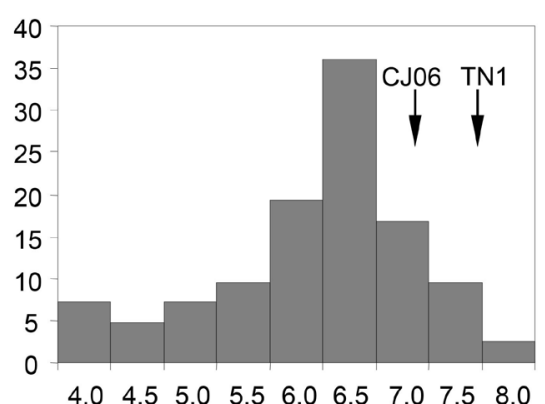

Grain length after cutting hull (mm)
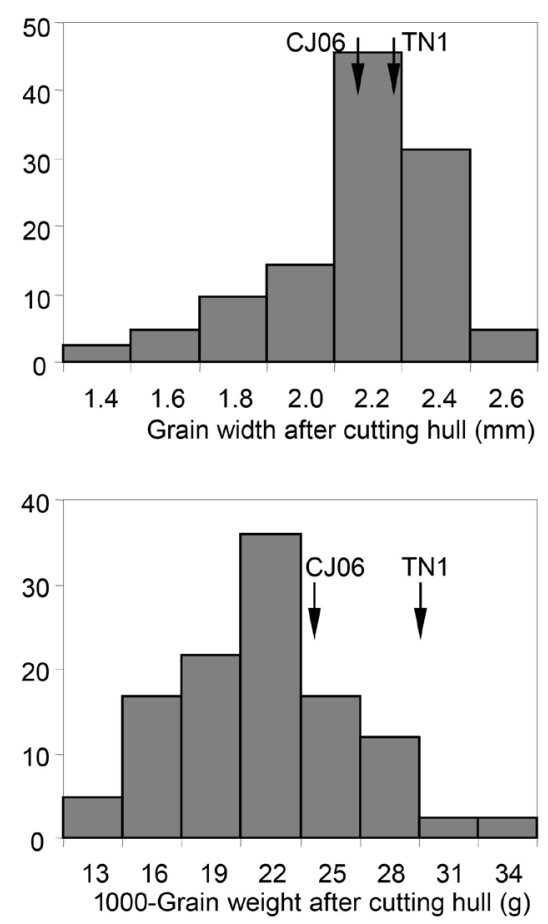

Figure 1. Distribution of grain shape and weight with and without cutting the husk in doubled haploid population. Grain length (A); width (B); and weight (C). 


\section{Phenotypic properties in the DH population}

All 120 lines in the $\mathrm{DH}$ population were detected in all six traits. Most lines exhibited elongated grain length and narrowed grain width. Because of the change of grain shape, grain weight also changed correspondingly. There were six kinds of situations (shown in Table 2). There was no line that showed shorter grain length and broader grain width. After cutting the husk, there were 85 lines with increased grain weight. Among these, 67 lines displayed elongated grain length and narrowed width and 18 lines showed increased grain length and width. Apart from the increased grain weight, 35 lines exhibited decreased grain weight. Among these, most showed elongated grain length and narrowed width. These results indicated that the rice husk usually suppresses rice filling.

\begin{tabular}{|c|c|c|c|}
\hline Grain length & Grain width & Grain weight & No. of lines \\
\hline+ & + & + & 18 \\
\hline+ & - & + & 67 \\
\hline+ & - & - & 27 \\
\hline- & - & - & 8 \\
\hline- & + & + & 0 \\
\hline- & + & - & 0 \\
\hline
\end{tabular}

$(+)$ Dates were increased after cutting the husk of rice; (-) dates were decreased after cutting the husk of rice.

\section{Correlation analysis of grain shape and weight-related traits}

Correlation analysis (Table 3) suggested that KGW exhibited a significant positive correlation to other traits, especially $G L$ and grain width (GW). It was not difficult to understand that KGW depended on GL and GW, with the correlation coefficients 0.7874 and 0.7722 . Similarly, the correlation coefficients among KGWC, GLC, and the grain width in cutting husk (GWC) showed a high significance. However, KGWC had no correlation with GL and GW. In addition, GLC and GWC showed a significant positive correlation to that without cutting the husk. Altogether, these results demonstrated that grain shape had a connection between that before and after cutting the husk. Nevertheless, grain weight after cutting the husk did not have a significant correlation to the grain shape before cutting the husk.

Table 3. Correlation analysis of grain shape and grain weight.

\begin{tabular}{lllll}
\hline & GLC & GWC & KGWC & GL \\
\hline GWC & $0.8454^{* *}$ & & & \\
KGWC & $0.7526^{* *}$ & $0.7964^{* *}$ & & \\
GL & $0.4362^{*}$ & $0.4125^{\star}$ & 0.2781 & $0.7388^{* *}$ \\
GW & $0.4747^{*}$ & $0.3805^{*}$ & 0.3413 & $0.7874^{* *}$ \\
KGW & $0.621^{* *}$ & $0.5697^{* *}$ & $0.6206^{* *}$ & $0.7722^{* *}$ \\
\hline
\end{tabular}

*,**Represent significant at the level 5 and $1 \%$, respectively. For abbreviations, see Table 1.

\section{QTL analysis for grain shape and weight}

Twenty-nine QTLs for grain shape and weight were detected in the DH population (Table 4; Figure 2). These distributed on chromosomes 1, 2, 3, 6, 8, 9, 10, and 12. Of these QTLs, 
six for GL were detected on chromosomes 1, 2, 3, 8, 9, and 10, and were named qGL1, qGL2, qGL3, qGL8, qGL9, and qGL10, respectively. These QTLs had the LOD score of 2.38 to 3.02 and explained variation of 9.96 to $18.43 \%$. Among them, a major QTL for GL was qGL8, which located in the interval of RM1376 and 4085, on chromosome 8 at the position $26.3 \mathrm{cM}$, with the highest LOD value and $18.43 \%$ phenotypic variation. The loci qGL2, qGL8, and qGL9 showed a negative additive effect, indicating that the allele from TN1 could increase grain length. However, after cutting the husk, there were seven QTLs for GLC. These had the LOD score of 2.41 to 4.72 and explained phenotypic variation of 7.56 to $19.82 \%$. A major QTL for GLC, qGLC8, was located in the interval of RM1111 and 310 on chromosome 8, with the highest LOD 4.72 and explained phenotypic variation (19.82\%). All of the QTLs exhibited a negative additive effect, with the exception of qGLC10. For example, the additive effect of qGLC8 was -1.6873 , indicating that the allele from TN1 could increase grain length by $1.6873 \mathrm{~mm}$ after cutting the husk.

Table 4. QTL analysis for grain shape and weight in the doubled haploid population.

\begin{tabular}{|c|c|c|c|c|c|c|c|}
\hline Traits & Locus & Position (cM) & Chr. & Marker interval & LOD & $\mathrm{H}^{2}(\%)$ & Additive \\
\hline \multirow[t]{6}{*}{$\mathrm{GL}$} & $q G L 1$ & 121.6 & 1 & RM1297-RM1061 & 2.61 & 12.54 & 0.7844 \\
\hline & $q G L 2$ & 75.0 & 2 & RM341-RM263 & 2.45 & 13.92 & -0.9437 \\
\hline & $q G L 3$ & 94.9 & 3 & RM6266-RM2334 & 2.38 & 13.92 & 0.8395 \\
\hline & $q G L 8$ & 26.3 & 8 & RM1376-RM4085 & 3.02 & 18.43 & -0.8197 \\
\hline & $q G L 9$ & 72.1 & 9 & RM242-RM278 & 2.88 & 12.76 & -0.7941 \\
\hline & qGL10 & 11.7 & 10 & RM5271-RM216 & 2.47 & 9.96 & 0.737 \\
\hline \multirow[t]{5}{*}{ GW } & $q G W 2$ & 75.0 & 2 & RM341-RM263 & 3.15 & 15.46 & -0.6194 \\
\hline & $q G W 3$ & 20.3 & 3 & RM489-RM545 & 4.70 & 21.18 & 0.978 \\
\hline & $q G W 6$ & 56.3 & 6 & RM527-RM3 & 2.8 & 13.97 & -0.576 \\
\hline & $q G W 8$ & 35.7 & 8 & RM4085-RM1111 & 3.33 & 15.90 & -0.6082 \\
\hline & $q G W 10$ & 11.7 & 10 & RM5271-RM216 & 2.48 & 10.84 & 0.533 \\
\hline \multirow[t]{5}{*}{ KGW } & $q G W T 1$ & 116.5 & 1 & RM1232-RM1297 & 2.07 & 8.02 & 3.45 \\
\hline & $q G W T 2$ & 75.0 & 2 & RM341-RM263 & 4.03 & 21.67 & -5.64 \\
\hline & qGWT6 & 56.3 & 6 & RM527-RM3 & 4.26 & 15.04 & -4.75 \\
\hline & qGWT8 & 26.3 & 8 & RM1376-RM4085 & 5.36 & 18.77 & -5.25 \\
\hline & qGWT10 & 11.7 & 10 & RM5271-RM216 & 2.89 & 11.00 & 4.28 \\
\hline \multirow[t]{7}{*}{ GLC } & $q G L C 1$ & 20.2 & 1 & RM5302-RM1 & 2.55 & 7.56 & -1.058 \\
\hline & $q G L C 2$ & 75.0 & 2 & RM341-RM263 & 2.49 & 10.97 & -1.2851 \\
\hline & qGLC3 & 122.8 & 3 & RM504-RM426 & 2.60 & 10.45 & -1.309 \\
\hline & $q G L C 6$ & 56.3 & 6 & RM527-RM3 & 2.43 & 11.54 & -1.293 \\
\hline & qGLC8 & 36.0 & 8 & RM1111-RM310 & 4.72 & 19.82 & -1.6873 \\
\hline & $q G L C 10$ & 11.7 & 10 & RM5271-RM216 & 2.41 & 9.11 & 1.209 \\
\hline & $q G L C 12$ & 80.0 & 12 & RM270-RM17 & 2.45 & 9.63 & -1.169 \\
\hline \multirow[t]{2}{*}{ GWC } & $q G W C 8$ & 36.0 & 8 & RM1111-RM310 & 2.97 & 26.2 & -0.3808 \\
\hline & $q G W C 10$ & 11.7 & 10 & RM5271-RM216 & 2.49 & 16.7 & 0.321 \\
\hline \multirow[t]{4}{*}{ KGWC } & qGWTC2 & 75.0 & 2 & RM341-RM263 & 2.80 & 12.81 & -5.24 \\
\hline & qGWTC3 & 122.8 & 3 & RM504-RM426 & 2.51 & 13.20 & -5.53 \\
\hline & qGWTC8 & 36.0 & 8 & RM1111-RM310 & 3.96 & 21.65 & -6.65 \\
\hline & qGWTC12 & 80.0 & 12 & RM270-RM17 & 2.60 & 17.03 & -5.86 \\
\hline
\end{tabular}

For abbreviations, see Table 1.

Seven QTLs for grain width with and without cutting the husk (GW and GWC) were identified. Among them, there were five for GW and two for GWC. These QTLs distributed on chromosome 2, 3, 6, 8 and 10, with the LOD score of 2.48 to 3.33 and explained phenotypic variation of 10.84 to $26.2 \%$. For GW, the QTL with the highest LOD value was qGW3, which was 
located in the interval of RM489-RM545 on chromosome 3 and explained phenotypic variation of $21.18 \%$. Moreover, the additive effect of qGW3 was 0.978 , which suggested that the allele from CJ06 could play an important role in grain width. Two QTLs for GWC, named qGWC8 and qGWC10, were mapped on chromosomes 8 and 10, with explained variations of 26.2 and $16.7 \%$, respectively. In addition, both qGW10 and qGWC10 were mapped in the same interval of RM5271RM216 on chromosome 10.

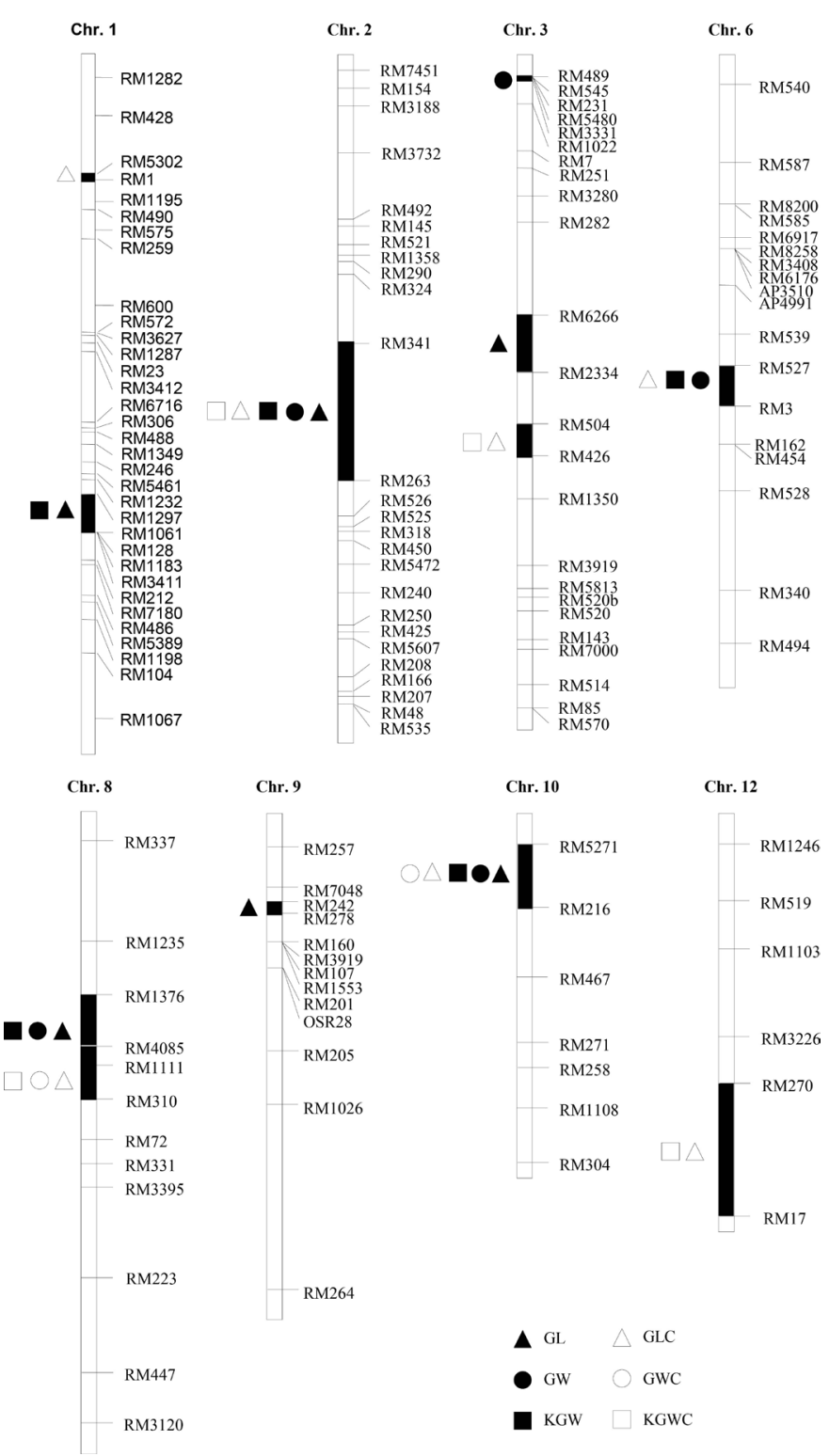

Figure 2. Chromosomal locations of QTLs for grain shape and weight with and without cutting the husk on the linkage map. For abbreviations, see Table 1. 
There were five QTLs, qKGW1, qKGW2, qKGW6, qKGW8 and qKGW10, for grain weight without cutting the husk (KGW). These were detected on chromosomes 1, 2, 6, 8, and 10, respectively, with LOD values ranging from 2.07 to 5.36 and the phenotypic variance explained from 8.02 to $21.67 \%$. qKGW8 was identified between RM1376 and RM4085 on chromosome 8, and can explain phenotypic variance of $18.77 \%$ with the LOD value of 5.36. qKGW6, another major QTL for KGW, mapped in the interval of RM527-RM3, and can explain phenotypic variance of 15.04. The allele of qKGW6 and qKGW8 from TN1 can increase the grain weight for 0.0475 and 0.0525 , respectively. After cutting the husk, four QTLs for KGWC were anchored on chromosomes 2, 3, 8, and 10, with the LOD value from 2.6 to 3.96 and the phenotypic variance from 12.81 to $21.65 \%$. One QTL for KGWC, qKGWC8, was located in the interval of RM1111-RM310 on chromosome 8. The allele from TN1 increased the grain weight by approximately $6.65 \mathrm{~g}$. In addition, the additive effect of all QTLs for KGWC exhibited negative value, which suggested that the alleles from TN1 play a great role in increased grain weight.

\section{DISCUSSION}

Rice husk has two known functions. First, it provides mechanical protection to rice filling. Second, during rice filling, the husk maintains humidity, preventing water loss from brown rice grains; blocks ultraviolet light; and ensures the smooth development of rice grain (Zhou et al., 2002). In our study, after hulling at the following time, 8 lines showed decreased grain length and width, which led to decreased grain weight. Furthermore, 27 lines also exhibited reduced grain weight, which may be due to the water loss or the affection of grain development after cutting the husk. Rice husk could limit grain development and grain filling could be suppressed by rice husk size and shape. Table 1 showed that most of the lines increased grain weight after cutting the husk. This suggests that rice husk was essential for grain development, or that the husk could hinder grain filling.

It is known that grain weight is dependent on grain shape; this was reconfirmed in the current study. Correlation analysis showed a tight connection between KGW and grain shape (GL and GW). The same results were observed among KGWC, GLC and GWC. However, no correlation was detected between KGWC and grain shape without husk removing (GL and GW), which indicated that GL and GW were not necessary for the formation of grain weight with cutting the husk. This finding suggests that the rice husk have great limitation to grain development in the DH population derived from a cross between CJ06 and TN1.

In this study, we identified 29 QTLs for rice grain shape- and weight-related traits distributing in 10 different intervals. Many intervals clustered two or more QTLs, suggesting that there may be connections among some traits. Identified QTLs showed a certain degree of specificity; some loci contributed to several traits; and some specifically contributed to a single trait. For example, the interval of RM341-RM263 on chromosome 2 was detected both under the two conditions including qGL, qGW, qKGW, qGLC, and qKGWC, whereas the QTL mapped in the interval of RM489-RM545 on chromosome 3 was found only in GL. In addition, the interval of RM1297-RM1061 was detected in traits with intact husk. Some exclusive QTLs for traits in huskremoved conditionsalso be detectedin the interval of RM504-RM426. All these results indicated that the genetic mechanisms to regulate grain shape and weight differed under the conditions of normal growth and husk removal.

Many QTLs for grain shape and weight had been previously reported; some were located in the region detected in this study. GL3.1, which encodes a Ser/Thr family protein phosphatase, 
accelerates cell division and result in longer grains and a higher yield (Qi et al., 2012). The gene was located in the interval of RM6266-RM2334, and overlapped the region of qGL3. In addition, two genes that have been previously associated with grain width, GW6a and TGW6, were anchored in the region from RM527 toRM3in our study (Ishimaru et al., 2013; Song et al., 2015). Li et al. (2003) reported a QTL for grain length, named qGL-6, located in the same interval of RM527RM3. In this study, this interval on chromosome 6 harbored three QTLs, named qGW6, qKGW6 and qGLC6, involved in the formation of grain length, width and weight. Therefore, the overlapped intervals can verify the results of QTL analysis in our study, to a certain extent. Some novel QTLs were also detected. For instance, the interval of RM270-RM17 was not detected without cutting the husk, but the QTLs qGLC12 and qKGWC12 were anchored to the RM270-RM17 interval on chromosome 12, which affected the grain length and weight after cutting the husk. These results suggest that this interval played an important role in grain development without the husk. It is reported that the protein phosphatase with Kelch-like repeat domain (PPKL) can regulate grain length, such as GL3.1/OsPPKL1, OsPPKL2 and OsPPKL3 (Zhang et al., 2012). Interestingly, OsPPKL3 was anchored in the interval of RM270-RM17, and regulated the grain length and grain weight. Therefore, OsPPKL3 may be responsible for qGLC12 and qKGWC12; further research is underway to verify this conclusion. All of the overlapped regions for grain shape and weight suggested a universal regulatory mechanism of grain development when grain filling.

Rice husk is an important component of the grain and provides grain filling and fixes grain shape. Grain shape plays a critical role in rice yield, but giant rice grain has not been widely utilized in rice breeding owing to the grain plumpness. In this study, we conducted a comparison of QTL analysis under two conditions to explore the genetic basis of grain shape and weight regulation. The results in this study can be beneficial to rice breeding by improving grain plumpness of giant rice grain and increasing rice yield. The QTL analysis herein provides a theoretical basis to grain filling and growth regulation.

\section{ACKNOWLEDGMENTS}

Research supported by grants from the Ganpo " 555 " Talents Project and from the Science Foundation of Jiangxi Province (grant \#20151BAB204024).

\section{REFERENCES}

Ashikari M, Wu J, Yano M, Sasaki T, et al. (1999). Rice gibberellin-insensitive dwarf mutant gene Dwarf1 encodes the alphasubunit of GTP-binding protein. Proc. Natl. Acad. Sci. U. S. A. 96: 10284-10289.

Hong Z, Ueguchi-Tanaka M, Umemura K, Uozu S, et al. (2003). A rice brassinosteroid-deficient mutant, ebisu dwarf (d2), is caused by a loss of function of a new member of cytochrome P450. Plant Cell 15: 2900-2910.

Huang R, Jiang L, Zheng J, Wang T, et al. (2013). Genetic bases of rice grain shape: so many genes, so little known. Trends Plant Sci. 18: 218-226.

Ishimaru K, Hirotsu N, Madoka Y, Murakami N, et al. (2013). Loss of function of the IAA-glucose hydrolase gene TGW6 enhances rice grain weight and increases yield. Nat. Genet. 45: 707-711.

Kitagawa K, Kurinami S, Oki K, Abe Y, et al. (2010). A novel kinesin 13 protein regulating rice seed length. Plant Cell Physiol. 51: 1315-1329.

Li F, Liu WB, Tang JY, Chen JF, et al. (2010). Rice DENSE AND ERECT PANICLE 2 is essential for determining panicle outgrowth and elongation. Cell Res. 20: 838-849.

Li Y, Fan C, Xing Y, Jiang Y, et al. (2011). Natural variation in GS5 plays an important role in regulating grain size and yield in rice. Nat. Genet. 43: 1266-1269.

Li ZF, Wan JM, Xia JF and Zhai HQ (2003). Mapping quantitative trait loci underlying appearance quality of rice grains (Oryza 
sativa L.). Yi Chuan Xue Bao 30: 251-259.

Ma J, An YP, Sun JC and Wang CF (2009). Effect of cutting hull on growth of Ningxia brown rice. Acta Agric. Bor-occ. Sin. 18: 71-75.

Mao H, Sun S, Yao J, Wang C, et al. (2010). Linking differential domain functions of the GS3 protein to natural variation of grain size in rice. Proc. Natl. Acad. Sci. U. S. A. 107: 19579-19584.

McCouch SR (2008). Gene nomenclature system for rice. Rice 1: 72-84.

Miura K, Ashikari M and Matsuoka M (2011). The role of QTLs in the breeding of high-yielding rice. Trends Plant Sci. 16: 319-326.

Qi P, Lin YS, Song XJ, Shen JB, et al. (2012). The novel quantitative trait locus GL3.1 controls rice grain size and yield by regulating Cyclin-T1;3. Cell Res. 22: 1666-1680.

Segami S, Kono I, Ando T, Yano M, et al. (2012). Small and round seed 5 gene encodes alpha-tubulin regulating seed cell elongation in rice. Rice 5: 4.

Song XJ, Huang W, Shi M, Zhu MZ, et al. (2007). A QTL for rice grain width and weight encodes a previously unknown RINGtype E3 ubiquitin ligase. Nat. Genet. 39: 623-630.

Song XJ, Kuroha T, Ayano M, Furuta T, et al. (2015). Rare allele of a previously unidentified histone $\mathrm{H} 4$ acetyltransferase enhances grain weight, yield, and plant biomass in rice. Proc. Natl. Acad. Sci. U. S. A. 112: 76-81.

Takeda S and Matsuoka M. (2008). Genetic approaches to crop improvement: responding to environmental and population changes. Nat. Rev. Genet. 9: 444-457.

Tan YF, Xing YZ, Li JX, Yu SB, et al. (2000). Genetic bases of appearance quality of rice grains in Shanyou 63, an elite rice hybrid. Theor. Appl. Genet. 101: 823-829.

Tanabe S, Ashikari M, Fujioka S, Takatsuto S, et al. (2005). A novel cytochrome P450 is implicated in brassinosteroid biosynthesis via the characterization of a rice dwarf mutant, dwarf11, with reduced seed length. Plant Cell 17: 776-790.

Unnevehr L, Duff B and Juliano BO (1992). Consumer Demand for Rice Grain Quality: Terminal Report of IDRC Projects National Grain Quality (Asia) and International Grain Quality Economics (Asia): Int. Rice Res. Inst.

Wang D, Zhu J, Li Z and Paterson A (1999). Mapping QTLs with epistatic effects and QTL x environment interactions by mixed linear model approaches. Theor. Appl. Genet. 99: 1255-1264.

Wang E, Wang J, Zhu X, Hao W, et al. (2008). Control of rice grain-filling and yield by a gene with a potential signature of domestication. Nat. Genet. 40: 1370-1374.

Wang S, Wu K, Yuan Q, Liu X, et al. (2012). Control of grain size, shape and quality by OsSPL16 in rice. Nat. Genet. 44: 950-954.

Wang TY, Yao YL, Li YL and Cai JZ (1995). An inquiring into grain characters and their relations with grain weight in rice (Oryza sativa L.). Acta Agric. Sin. 5: 010.

Wang Y, Yamamoto Y and Yao Y (1998). effect of cultural conditions on grain weight and it's causes in rice. Acta Agric. Sin. 24: $280-290$.

Weng J, Gu S, Wan X, Gao H, et al. (2008). Isolation and initial characterization of GW5, a major QTL associated with rice grain width and weight. Cell Res. 18: 1199-1209.

Yamamuro C, Ihara Y, Wu X, Noguchi T, et al. (2000). Loss of function of a rice brassinosteroid insensitive1 homolog prevents internode elongation and bending of the lamina joint. Plant Cell 12: 1591-1606.

Yang Y, Rao Y, Xu J, Shao G, et al. (2014). Genetic analysis of sugar-related traits in rice grain. South Afr. J. Bot. 93: 137-141.

Zhang X, Wang J, Huang J, Lan H, et al. (2012). Rare allele of OsPPKL1 associated with grain length causes extra-large grain and a significant yield increase in rice. Proc. Natl. Acad. Sci. U. S. A. 109: 21534-21539.

Zhou XD, Wang YL, Sun LJ, Dong GC, et al. (2002). Effect of Cutting Hull on Growth of Brown Rice. J. Yangzhou Univ. (Agric. Life Sci. Edit.) 24: 41-49. 